\title{
Tratamiento de eventraciones gigantes con cirugía de separación de componentes más turn over flap aponeurótico
}

\author{
Kenneth Guler G. ${ }^{1,2}$, Juan Pablo Camacho M. ${ }^{1,2}$, Rodrigo Subiabre F. ${ }^{1,2}$, \\ Karina Vargas V. ${ }^{1,2}$ y Wilfredo Calderón O., ${ }^{1,2}$
}

'Servicio de Cirugía Hospital El Salvador. Santiago, Chile. 2Departamento de Cirugía Oriente, Universidad de Chile. Santiago, Chile.

Recibido el 6 de mayo de 2019 y aceptado para publicación el 16 de diciembre de 2019

Correspondencia a: Dr. Juan Pablo Camacho M jpcamachomartino@gmail.

\section{Giant ventral hernia treatment with components separation technique and aponeurotic turn over flap}

\begin{abstract}
Aim: To demonstrate the anatomy and application of the anterior rectus sheet in the repair of giant abdominal wall hernias through a classic component surgery plus a turn over flap. Materials and Method: Anatomic dissection with vascular and dynamic description of the anterior rectus sheet. Posterior application of the technique in patients with giant abdominal wall hernias. Clinical follow up of recurrence, local and systemic complications. Results: 8 patients were selected. The average age was 58.6 years old and the abdominal wall defect $19.6 \mathrm{~cm}$ wide. The technique could be applied in every patient without inconveniences. Only 1 patient had a complication (dehiscence and partial skin flap necrosis) that was successfully treated with resection and readvancement. No systemic complications nor mortality was described. Discussion: Giant abdominal wall hernias are a surgical challenge. Great and innovative advances have been made. New biological and synthetic materials have been developed, nevertheless they frequently involve great costs and complications related to them. Also, they do not adapt adequately to the dynamic required for the abdominal wall. Conclusion: This technical modification is useful, safe and accessible for the patients who present giant wall hernias. The complication rate is low and it's principally given from problems related to skin flap blood flow.
\end{abstract}

Key words: abdominal hernia; herniorrhaphy; perforator flap; rectus abdominis; fascia.

\section{Resumen}

Objetivo: Demostrar la anatomía y aplicación de la vaina anterior de los rectos para la reparación de defectos herniarios gigantes de la línea media abdominal mediante la cirugía de separación de componentes más la aplicación de un colgajo aponeurótico tipo turn over. Materiales y Método: Disección anatómica con descripción vascular y dinámica de la vaina anterior de los rectos. Posterior aplicación de la técnica en pacientes con hernias gigantes de la línea media. Seguimiento clínico de recidiva y complicaciones locales como sistémicas. Resultados: 8 pacientes fueron seleccionados. La edad promedio fue de 58,6 años y el tamaño del defecto herniario $19,6 \mathrm{~cm}$. En todos los pacientes se pudo aplicar la técnica sin inconvenientes. Sólo 1 paciente presentó una complicación local (dehiscencia y necrosis parcial del colgajo cutáneo) que se manejó con resección y reavance sin incidentes. No se describen complicaciones sistémicas ni mortalidad. Discusión: Las hernias abdominales gigantes y con pérdida a derecho de domicilio son un desafío quirúrgico. Se han desarrollado importantes avances con abordajes quirúrgicos innovadores. Nuevos materiales biológicos y sintéticos se han convertido en una parte integral del arsenal quirúrgico, sin embargo, involucran muchas veces asumir grandes costos y complicaciones propias a los materiales utilizados, además, de no cumplir con la adaptación dinámica adecuada de la pared requerida. Conclusión: Esta modificación técnica es segura, útil y accesible para los pacientes con eventraciones gigantes. La tasa de complicaciones es baja y está dada principalmente por problemas relacionados al sufrimiento de los colgajos cutáneos. Palabras clave: hernia abdominal; hernioplastía; colgajo perforante; recto abdominal; fascia. 


\section{Introducción}

Los defectos de la pared abdominal pueden clasificarse como congénitos o adquiridos, encontrándose dentro de estos últimos las hernias incisionales o eventraciones gigantes. Esta puede ser consecuencia de cirugías, traumas, infecciones masivas, necrosis por radiación o por la resección de tumores. Se entiende por hernia incisional grande, aquella cuyo anillo mide entre 10 y $15 \mathrm{~cm}$ en su diámetro máximo, y gigante, cuando sobrepasa los $15 \mathrm{~cm}^{1}$. Dentro de estos grupos existe un porcentaje que no logra el cierre completo del defecto aponeurótico posterior a la cirugía de separación de componentes (CSDC). En estos casos es necesario el uso de una malla que funcione como puente entre los bordes de la aponeurosis y a su vez tenga propiedades especiales que permitan su contacto con las asas intestinales. Las mallas de elección que se usan actualmente son las de tipo biológico ${ }^{2}$. La experiencia con estos materiales ha sido exitosa $a^{3-8}$, sin embargo, su valor comercial es elevado, restringiendo su uso. Ante la necesidad de dar solución a los pacientes que no pueden acceder a dicho material es que hace un par de años nuestro equipo desarrolló una técnica basada en la separación de componentes clásica más un turn-over flap aponeurótico de la hoja anterior del músculo recto, permitiendo el cierre de defectos mayores a $20 \mathrm{~cm}$. Esto se logra mediante tejido autólogo, sin tensión y sin la necesidad de utilizar mallas biológicas.

El objetivo de este trabajo es describir la técnica, mostrar el estudio en disección cadavérica y revisión de la literatura.

\section{Materiales y Método}

1. Estudio de la anatomía e irrigación vascular de la vaina anterior del recto abdominal en preparados cadavéricos fijados con tinción vascular mediante látex. Evaluación cualitativa y cuantitativa del movimiento de esta hoja desde lateral a medial.

2. Análisis de estrategia, con posterior diseño y descripción de la técnica.

3. Aplicación de la técnica en pacientes con eventraciones gigantes junto con medición de la presión intraabdominal (PIA) postoperatoria, descripción de complicaciones y resultados postoperatorios.

\section{Criterios de inclusión}

Hernias de la línea media con un anillo mayor o igual a $15 \mathrm{~cm}$.

\section{Criterios de exclusión}

Comorbilidades que impidan cualquier procedimiento quirúrgico. Hernias fuera de la línea media.

\section{Resultados}

\section{Estudio anatómico}

El estudio cadavérico demostró la factibilidad técnica de esta modificación. Se encontraron un adecuado número de perforantes hacia la línea media que permiten irrigar satisfactoria y plenamente la hoja anterior de la vaina del recto y, por lo tanto, se trataría de un tejido adecuadamente vascularizado. En cuanto a su movilización y avance hacia la línea media, pudimos observar que es factible de realizar, manteniendo su irrigación y respetando la línea de perforantes que emergen a $2 \mathrm{~cm}$ del borde medial de la línea alba. La longitud alcanzada es de $4 \mathrm{~cm}$ por lado aproximadamente (Figura 1).

\section{Descripción de la técnica}

La técnica se basa en la descripción clásica de la CSDC más una variación respecto al manejo de la vaina anterior de los rectos abdominales. El primer avance se logra con la disección de la línea semilunar bilateralmente y separación de los componentes musculares. Posterior a esto se procede a la sección de la vaina anterior de los rectos abdominales de lateral a medial, asegurando preservar las perforantes descritas. Luego se evierten ambas hojas aponeuróticas en la línea media. Finalmente, se instala una malla sintética sobre los músculos rectos como refuerzo. Lo denominamos "Turn Over Flap aponeurótico" (Figura 2).

\section{Aplicación clínica}

Un total de 8 pacientes fueron seleccionados. La edad promedio fue de 58,6 años y el tamaño del defecto herniario 19,6 cm. En todos los pacientes se realizó una evaluación preoperatorio clínica junto con laboratorio y tomografía computada de abdomen y pelvis con contraste (Tabla 1). Finalmente, se realizó evaluación preanestésica en los pacientes que lo requirieron. La disección en vivo confirmó los hallazgos realizados previamente en los estudios cadavéricos. En la totalidad de los pacientes se logró realizar la técnica descrita con afrontamiento libre de tensión en la línea media y refuerzo sobre el plano muscular. En todos los pacientes se instalaron drenajes aspirativos, los cuales fueron retirados al décimo día posterior a la cirugía. El tiempo operatorio promedio fue de $3,1 \mathrm{~h}$. El postoperatorio 


\section{ARTÍCULO ORIGINAL}

Figura 1. Disección anatómica cadavérica. Elevación hoja anterior de la vaina anterior del recto abdominal respetando perforantes a $2 \mathrm{~cm}$ de la línea media y avance hacia lado contralateral.

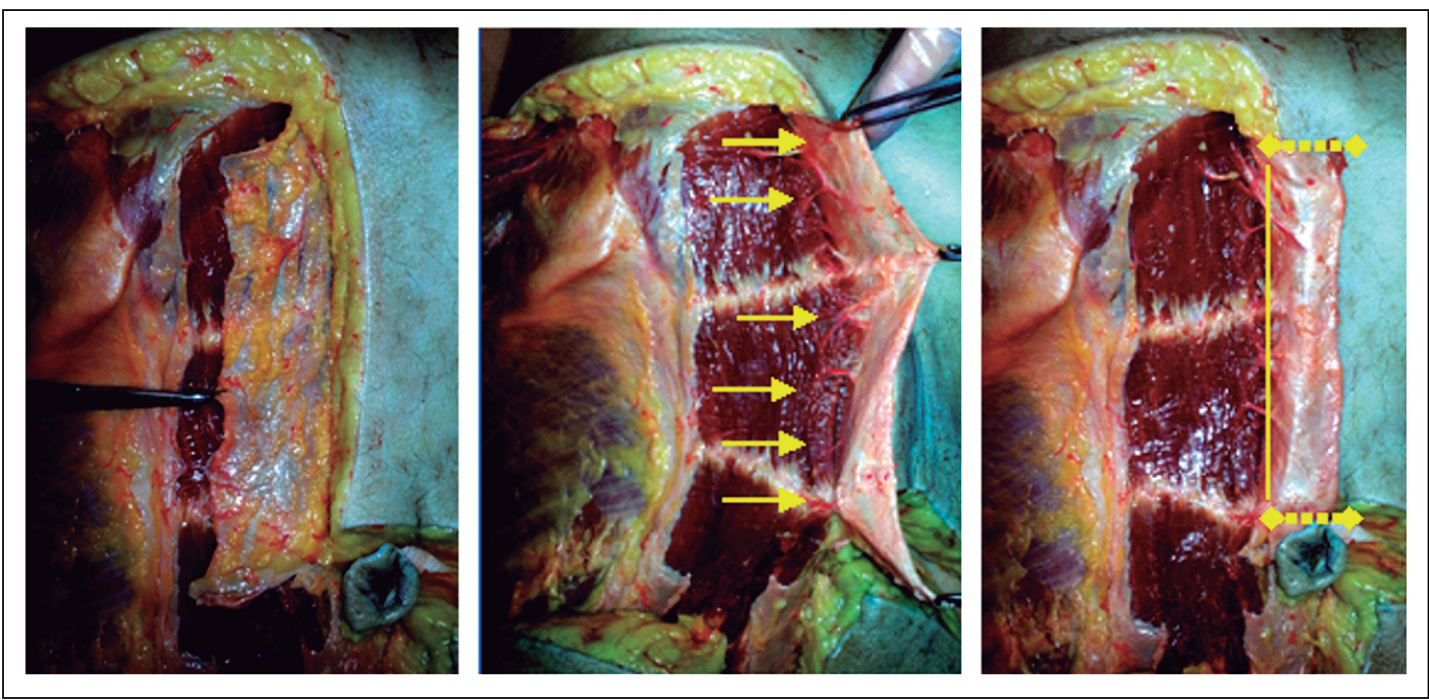

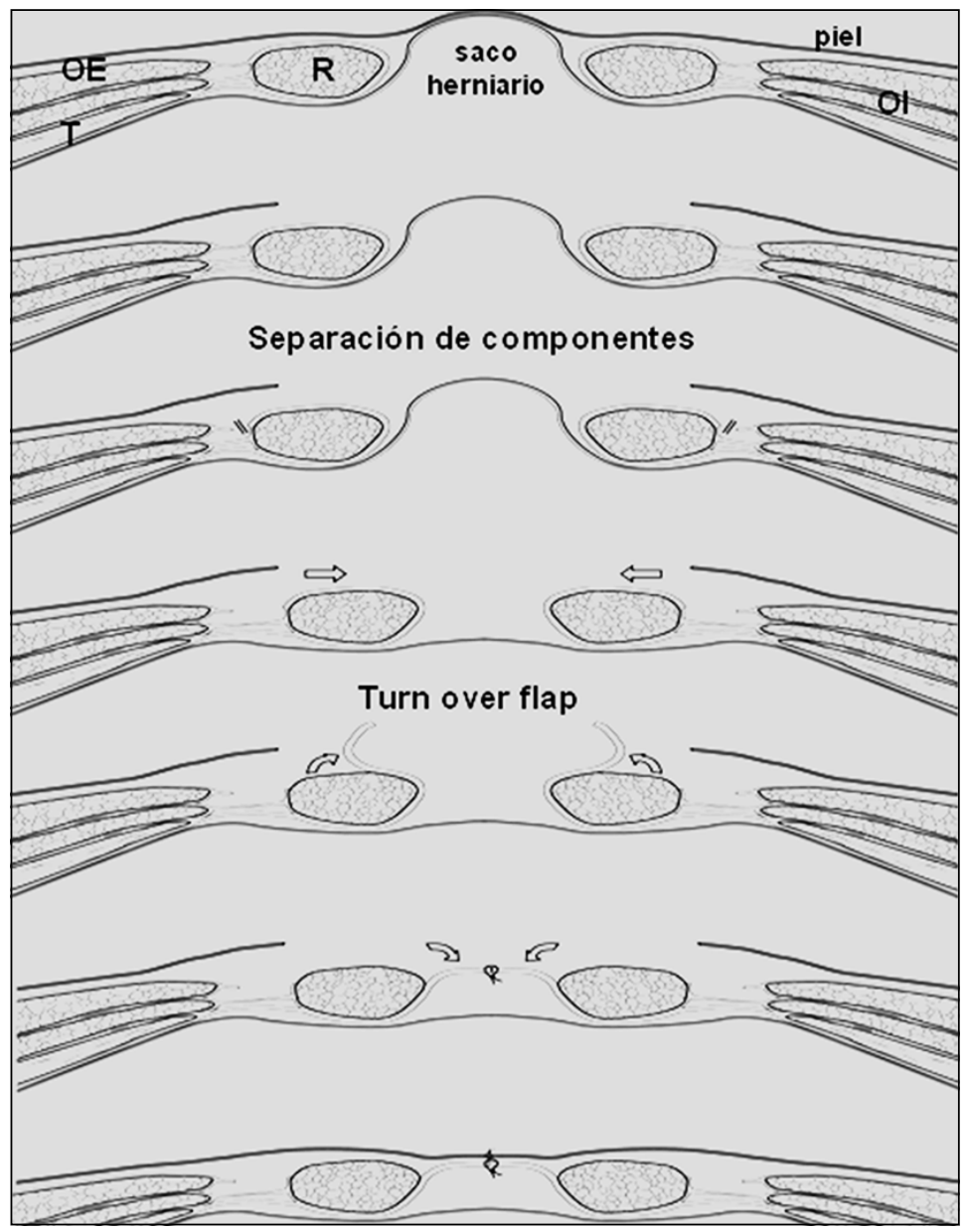

Figura 2. Esquema de técnica de separación de componentes más turn over flap aponeurótico. OE: Músculo oblicuo externo. OI: Músculo oblicuo interno. T: Músculo transverso. R: Músculo recto abdominal. inmediato se cumplió en unidad de cuidados intermedios donde se realizaron mediciones de la presión intraabdominal (PIA) mediante cateterismo vesical además de parámetros clínicos y laboratorio $(\mathrm{pH}$, equilibrio ácido-base, hipoxemia y diuresis). No hubo casos de síndrome compartimental, siendo el promedio de la PIA de v11 mmHg. El tiempo promedio de hospitalización fue de 16 días. Hubo un caso en el postoperatorio mediato de necrosis parcial del colgajo de pared abdominal correspondiente al último paciente de la serie que se manejó con resección, aseo y reafrontamiento. Destaca del mismo el antecedente de una cicatriz paramediana derecha. El seguimiento fue de 1 año sin tener registros de recidiva herniaria ni complicaciones locales a largo plazo. A continuación, mostramos algunos pacientes tratados con esta técnica y sus resultados postoperatorio.

\section{Discusión}

\section{Aspectos generales}

Varias condiciones contribuyen al desarrollo de las hernias incisionales, tales como: tipo de sutura, técnica de reparación y la tensión, cicatrización deficiente de heridas (a menudo asociada con la mala nutrición del paciente), infecciones, aumento de la presión intraabdominal (por ejemplo, debido a EPOC o uropatía obstructiva) o trastornos del tejido conectivo y alteraciones metabólicas ${ }^{1,2,9}$.

Fisiopatológica y clínicamente es importante destacar que la apertura parietal con sagitalización de los músculos rectos facilita la enucleación del conte- 
Tabla 1. Características pacientes evaluados y resultados postoperatorios

\begin{tabular}{|c|c|c|c|c|c|c|c|c|}
\hline Paciente $\left(\mathrm{N}^{\mathbf{0}}\right)$ & 1 & 2 & 3 & 4 & 5 & 6 & 7 & 8 \\
\hline Edad (años) & 72 & 74 & 62 & 56 & 75 & 44 & 45 & 41 \\
\hline Sexo & M & M & M & M & $\mathrm{F}$ & $\mathrm{F}$ & M & $\mathrm{F}$ \\
\hline Anillo (cm) & 20 & 20 & 20 & 17 & 20 & 22 & 20 & 18 \\
\hline Complicaciones & $\begin{array}{l}\text { Dehiscencia y necro- } \\
\text { sis parcial colgajo }\end{array}$ & & & & & & & \\
\hline Hospitalización (días) & 59 & 6 & 5 & 41 & 4 & 6 & 4 & 3 \\
\hline Recidiva & no & no & no & no & no & no & no & no \\
\hline
\end{tabular}

M: Masculino. F: Femenino. HTA: Hipertensión arterial. DM2 Diabetes mellitus 2. HPB: Hiperplasia prostática benigna. CC: Cardiopatía coronaria. GT: Gastrectomía total. LP: Laparostomía. PAG: Pancreatitis aguda grave.
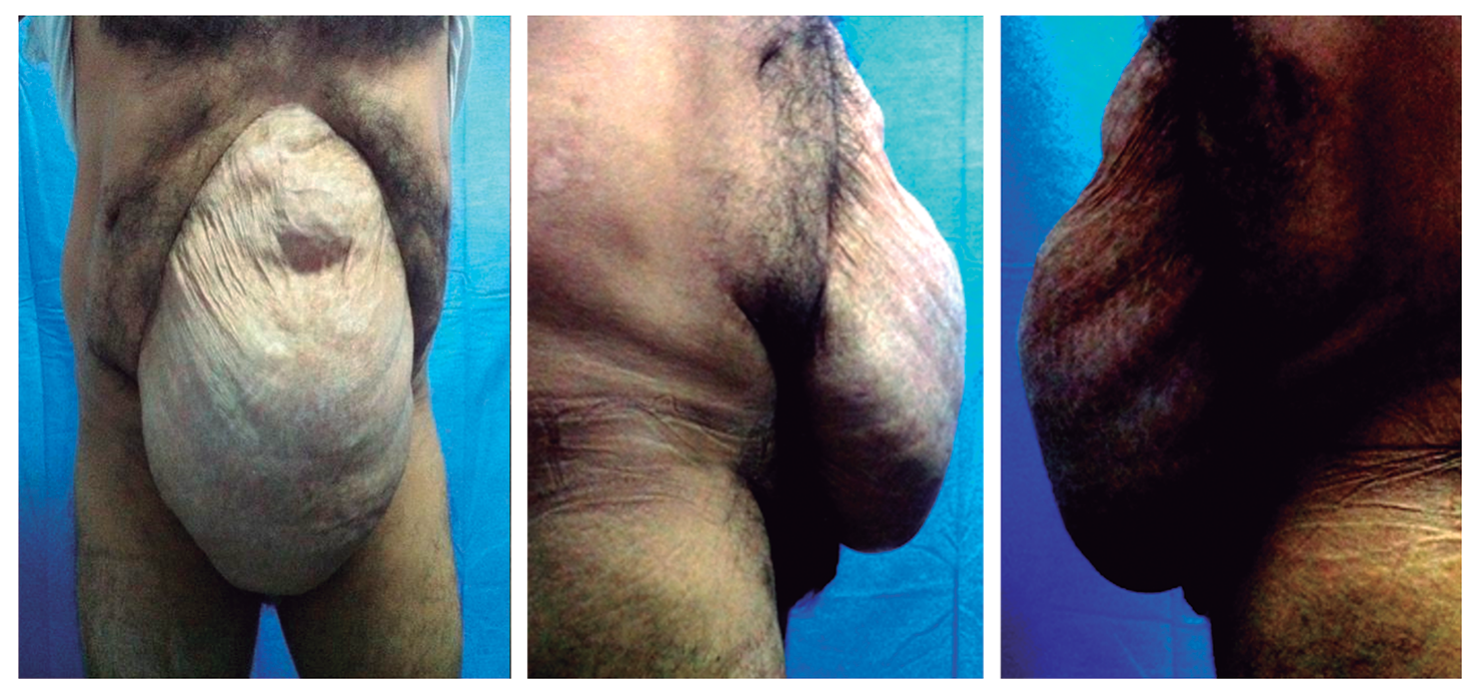

Figura 3. P4 Paciente con hernia incisional gigante. A: Vista frontal; B: Vista lateral.

nido abdominal hacia un "segundo abdomen", por lo tanto, la hernia se produce por la retracción a nivel de la unión de las vainas anteriores y posteriores más que por un defecto muscular. Posteriormente, esta alteración local evoluciona sistemáticamente, produciendo a nivel biomecánico una descoordinación entre pared abdominal, diafragma y pared abdominal, junto con una disminución PIA y atonía diafragmática progresiva. No obstante, el riesgo mayor radica en la reintegración de las vísceras a la cavidad abdominal, poniendo de nuevo la pared en tensión y sobre todo, aumentado súbitamente PIA sobre una mecánica abdominal y diafragmática ya alteradas $^{9-11}$. En cuanto a las metas de una reparación herniaria exitosa, se describen como objetivos para el cierre ideal de la pared abdominal ${ }^{11}$ :

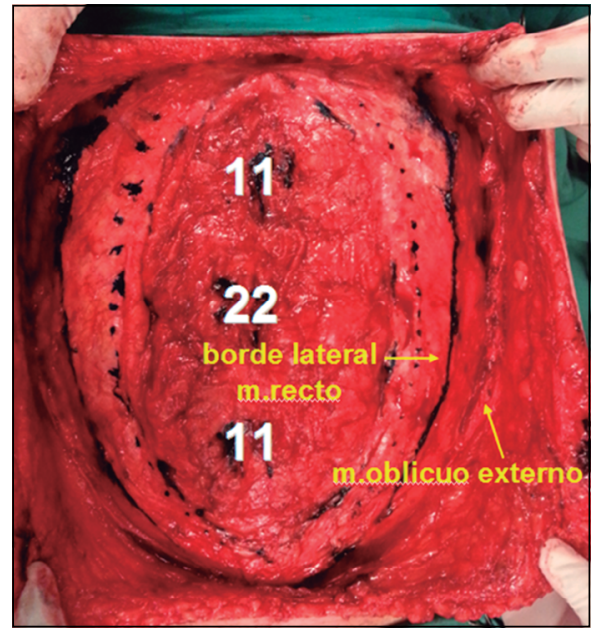

Figura 4. P4 Técnica de separación de componentes. Se observa el tamaño del defecto: $11 \mathrm{~cm}, 22 \mathrm{~cm}$ y $11 \mathrm{~cm}$. 
Figura 5. P1 Técnica Turn Over Flap aponeurótico.

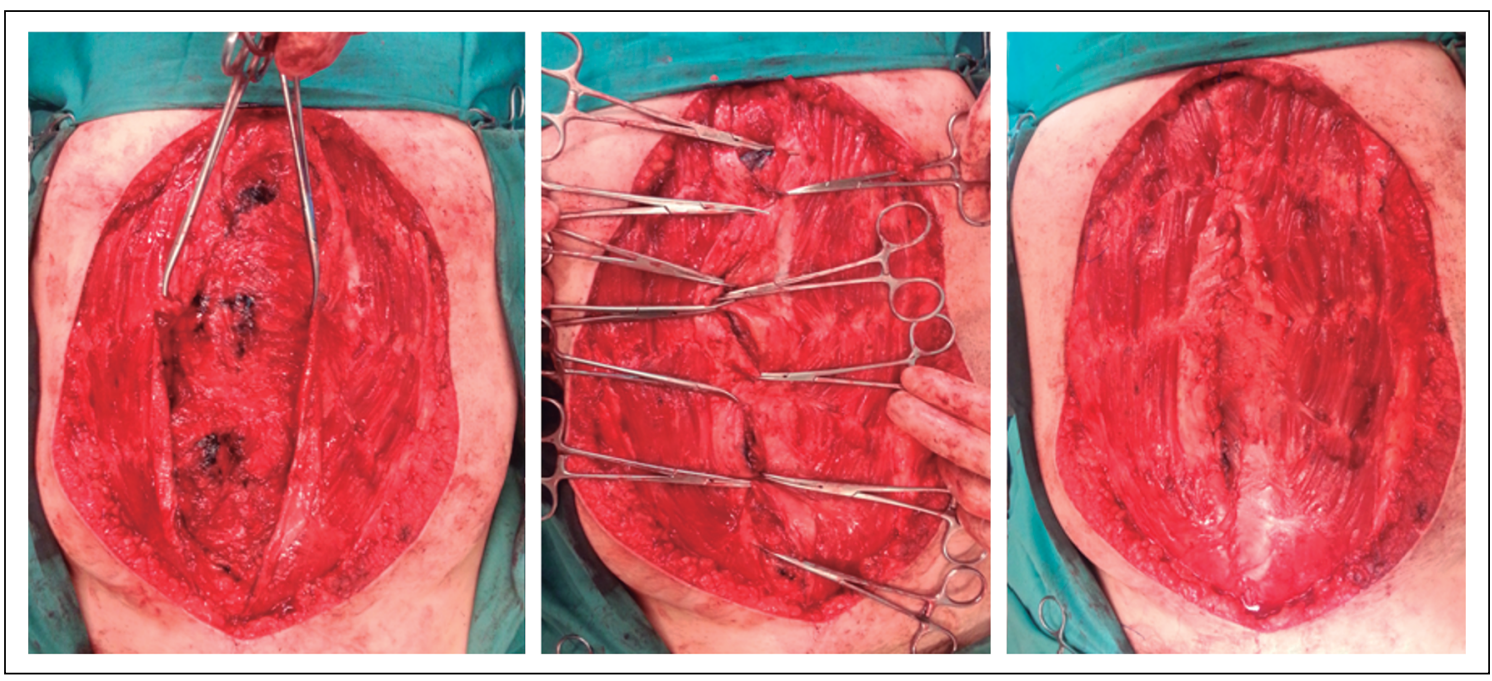

Figura 6. P4 Resultado a los 3 meses.

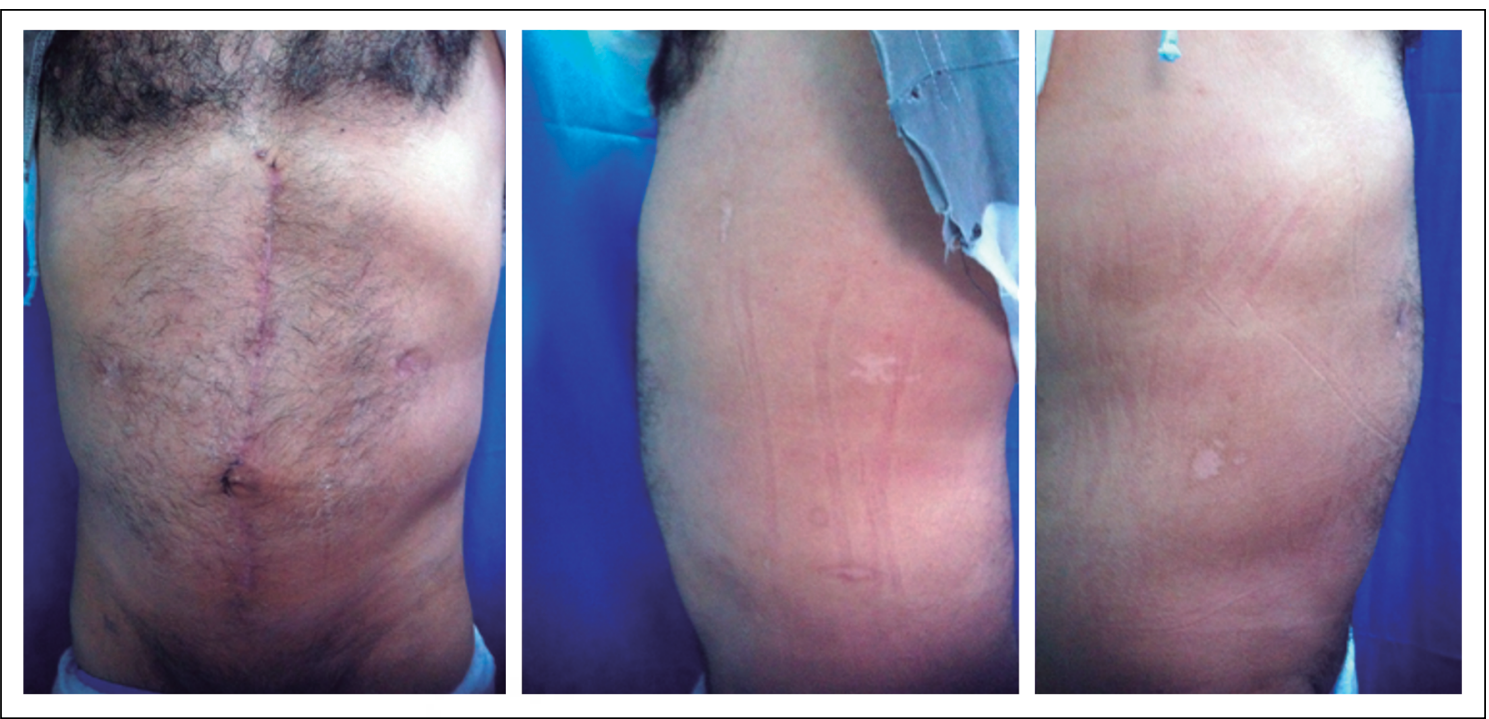

1. La prevención de eventración.

2. Incorporación de la pared abdominal.

3. Reparación libre de tensión.

4. Ofrecer apoyo muscular dinámico.

En cuanto al material ideal para su realización se establece que este sea: resistente, elástico, con mínima respuesta inflamatoria, resistente a infección, inclusión de tejido conectivo y vascularización y, por último, que produzca mínimas adherencias y obstrucción ${ }^{12}$. Las decisiones relativas a la técnica se basan en la evaluación del estado clínico general del paciente, la ubicación y el tamaño del defecto, la extensión de las capas implicadas y la etiología. Se han descrito múltiples técnicas para su corrección a lo largo de la historia, sin embargo, no existe consenso actual para un tratamiento óptimo único.

\section{Técnica de separación de componentes}

La CSDC es una técnica que se utiliza para proporcionar una cobertura adecuada a los defectos de la línea media de la pared abdominal, como es el caso de hernias ventrales de gran tamaño. Las primeras nociones de esta técnica fueron descritas por Donald Young quien por primera vez habla sobre la liberación del oblicuo externo en $1960^{11}$. Treinta años después, Ramírez describe y desarrolla la técnica como tal (SCDC) ${ }^{12}$. Este método utiliza colgajos musculares bilaterales, inervados y bipedi- 


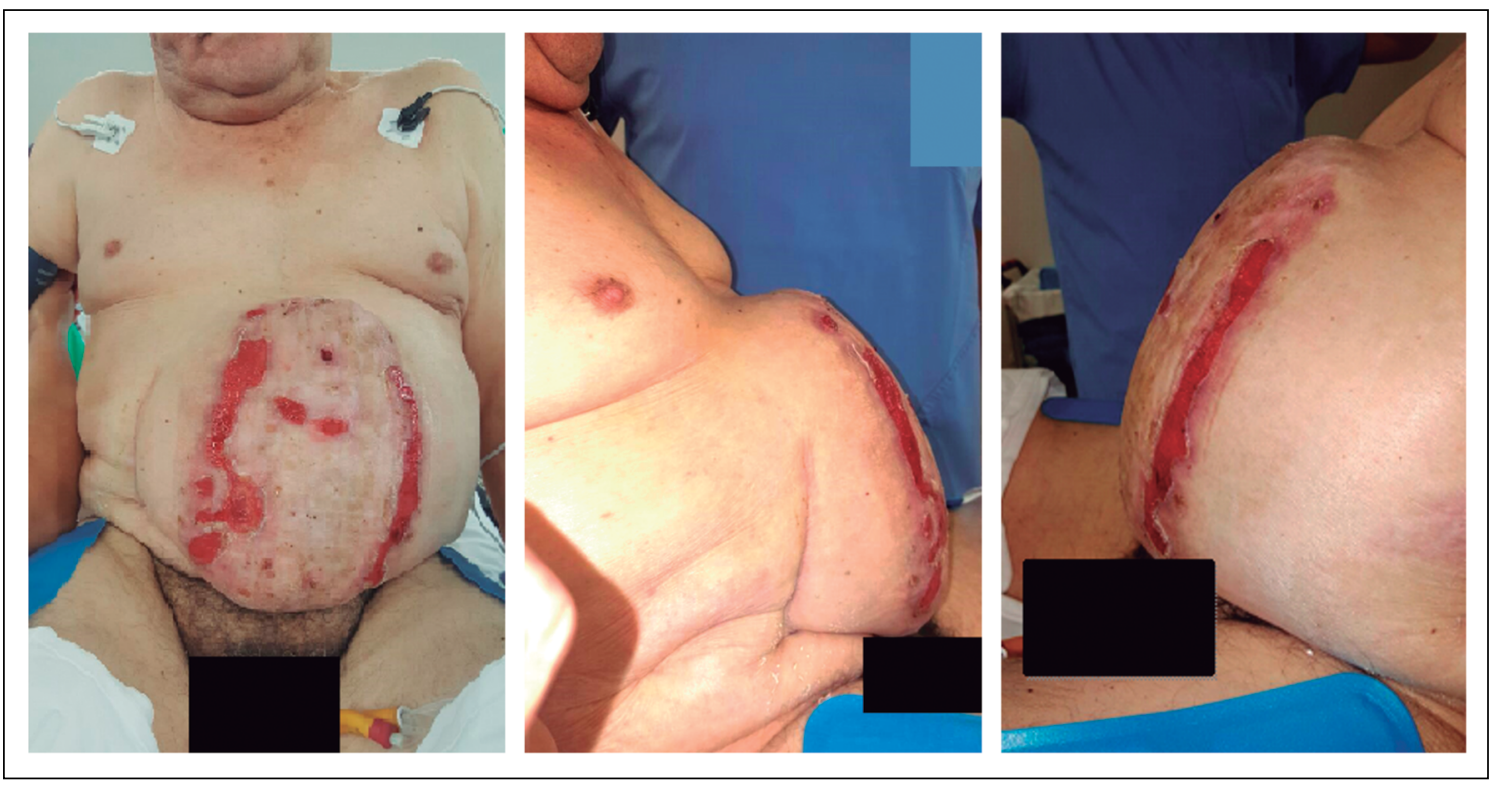

Figura 7. P1. Paciente con lesión ulcerativa de piel saco A: vista frontal; B: vista lateral. culados que son avanzados hacia la línea media para reconstruir defectos de la pared abdominal centrales. La técnica de separación de componentes representa un hito para las técnicas de transferencia de tejidos utilizados para reparar los defectos complejos de la línea media abdominal ${ }^{13}$. La técnica que se describió por primera vez expone la vaina anterior del músculo recto del abdomen, la aponeurosis del músculo oblicuo externo y la línea semilunar. La aponeurosis del oblicuo externo se secciona longitudinalmente lateral a la vaina del recto en una línea que se extiende desde el margen costal hasta el pubis. Se diseca el plano entre el músculo oblicuo externo y el músculo oblicuo interno lo más lateral posible. Se avanzan los colgajos hacia la línea media y se suturan creando una nueva línea blanca ${ }^{12-14}$.

De Vries Reilingh et al. en 2003, en su estudio realizado en 37 pacientes distribuidos aleatoriamente entre grupo 1: CSDC técnica clásica y grupo 2: Malla e-PTFE intraabdominal con un seguimiento 36 meses, describe diferencia estadísticamente significativa en tiempo operatorio $(1=113-2=183 \mathrm{~min})$ y reoperación $(10,5 \%$ - 38,8\%), resultados que se reafirman en posteriores estudios ${ }^{15}$. Posteriormente el 2007, el mismo grupo de autores realizaron un estudio retrospectivo donde reportaron similares tasas de recurrencia, hematoma, seroma y necrosis de la piel en un estudio controlado aleatorio que comparó complicaciones de la herida y tasa de recurrencia entre la reparación con malla intraperitoneal y la técnica de separación de componentes ${ }^{16}$. Tampoco el grupo con malla tuvo mayores tasas en lo que

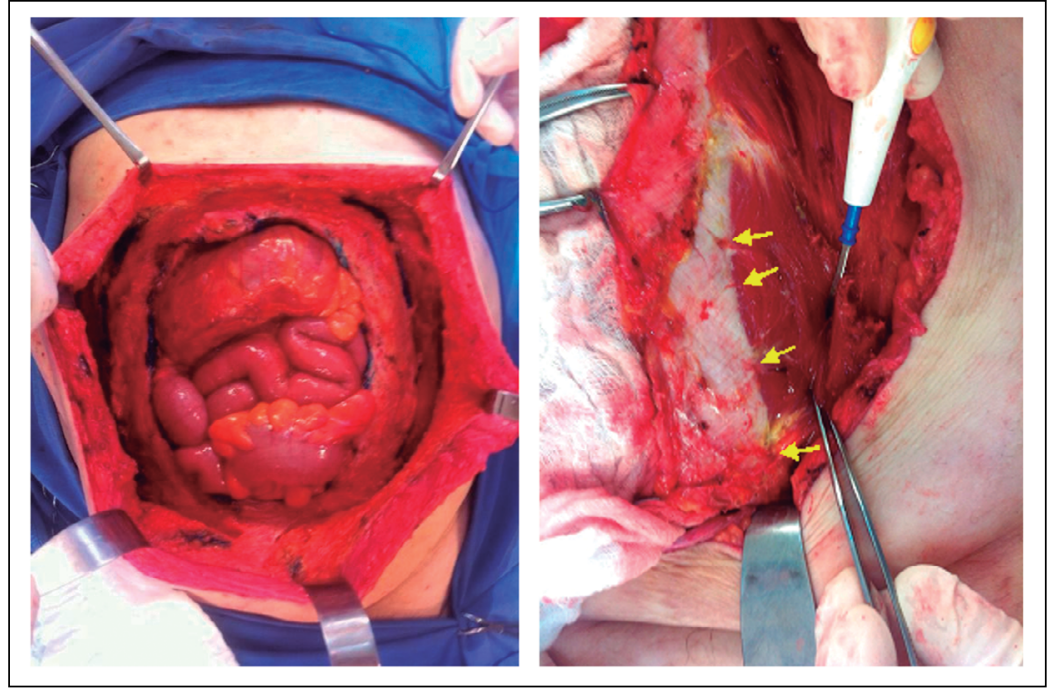

Figura 8. P1 Disección intraoperatoria con identificación y preservación de perforantes.

respecta a la infección de la herida que requiriera reintervención. Sin embargo, en el grupo con CSDC se registraron menos complicaciones catastróficas, tales como la formación de fístulas. Se cree que las complicaciones de la herida, relacionadas con CSDC, están relacionadas con la sección de las arterias perforantes del recto que irrigan la piel suprayacente cuando se crea el colgajo de piel para encontrar la línea semilunar. Por lo tanto, el suministro de sangre a los colgajos cutáneos abdominales es fundamental para la cicatrización de la herida ${ }^{17} \mathrm{y}$, 


\section{ARTÍ́CULO ORIGINAL}

sólo las perforantes que son absolutamente necesarias para la exposición se deberían ligar.

La separación de componentes endoscópica se ha descrito basándose en el mismo concepto de la preservación de las perforantes. La idea es liberar la aponeurosis del oblicuo externo endoscópicamente reduciendo el daño de los vasos perforantes y, por ende, de la piel ${ }^{18-20}$.

\section{Uso de mallas y subtipos biológicos}

Las mallas se dividen en dos grandes grupos: mallas sintéticas o protésicas y mallas biológicas o

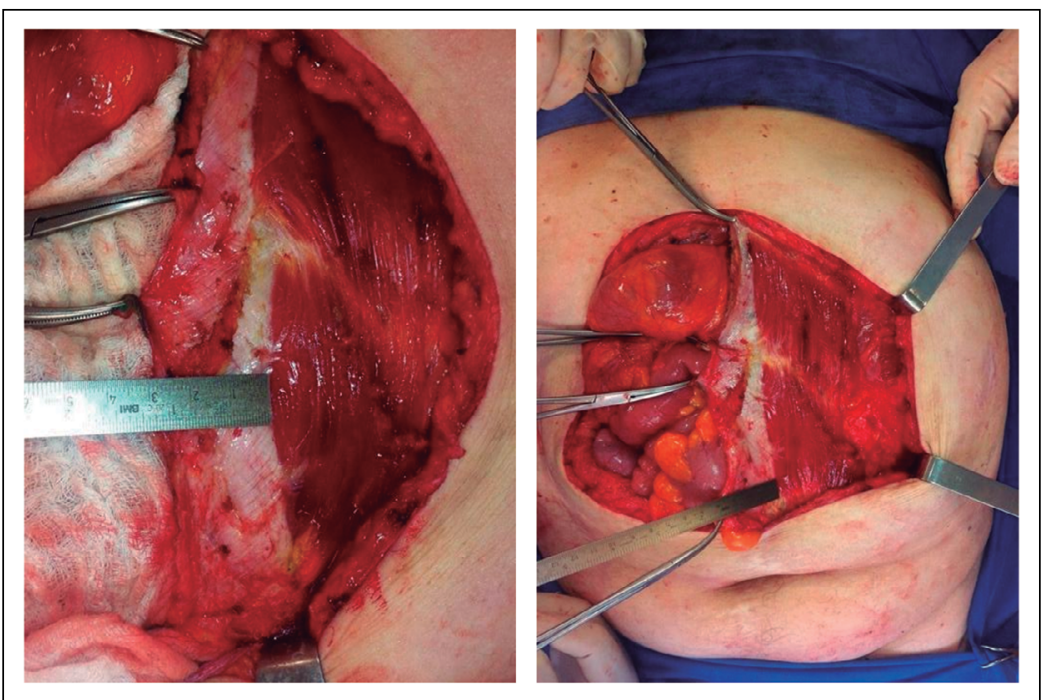

Figura 9. P1 Medición colgajo evertido (turn over flap) de vaina anterior de recto abdominal. bioprotésicas. Las mallas se utilizan comúnmente como soporte estructural o como puente para el tratamiento definitivo de la pared abdominal. El uso de mallas está relativamente contraindicado en los casos de infección de la herida, estrangulación herniaria y el contacto con el intestino ${ }^{5,20}$.

Las mallas sintéticas ofrecen alta resistencia a la tracción, sin embargo, se asocian a una respuesta inflamatoria crónica y a la formación de cicatrices que, posteriormente, promueven el crecimiento del tejido visceral en las porosidades de las mallas lo que puede conducir a complicaciones tales como adherencias, obstrucción intestinal y fístula enterocutánea ${ }^{5,20}$. Prótesis con características duales, absorbible y no absorbible, se han desarrollado con el fin de reducir las complicaciones antes mencionadas, sin embargo, no hay datos suficientes para apoyar su uso sobre las tradicionales.

En la última década, el uso de la matriz dérmica acelular se ha popularizado para el refuerzo de los tejidos blandos en la reconstrucción de la pared abdominal en las siguientes situaciones: como sustituto de colgajos, en reparaciones con malla protésica fallidas y en hernias complejas en las que no se aconseja malla sintética. Estos productos están constituidos de matrices dérmicas y submucosas derivadas de animales (xenoinjertos) o de humanos (aloinjertos) ${ }^{5}$ a remodelación del tejido huésped mientras mantiene la integridad mecánica. Se diferencian en que actúan a través de un proceso de regeneración celular en lugar de la formación de tejido cicatricial.

Entre las características de estas bioprótesis están

Figura 10. P1 Afrontamiento y posterior refuerzo con malla supra aponeurótica. Finalmente, cierre de piel con dos drenajes aspirativos. Nótese la cicatriz paramediana derecha.

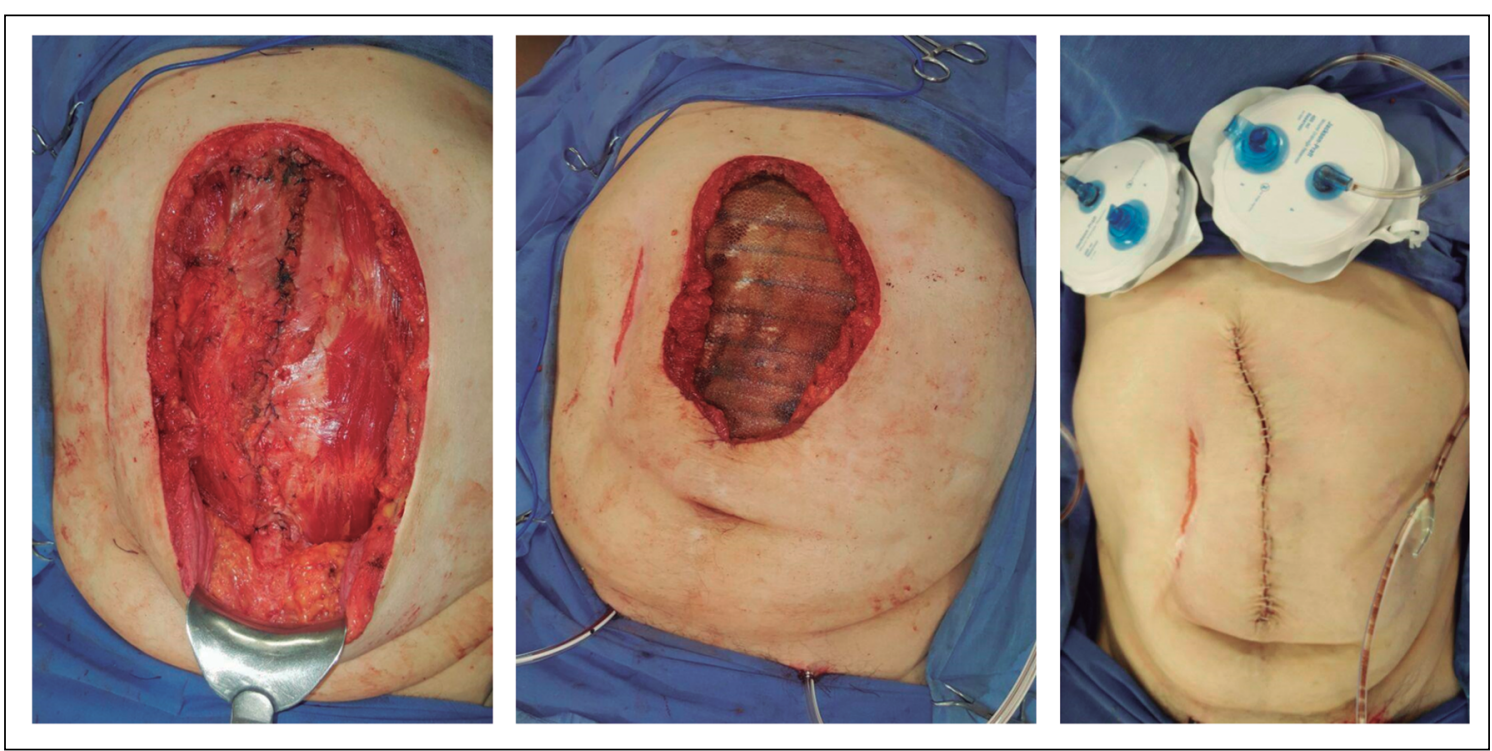




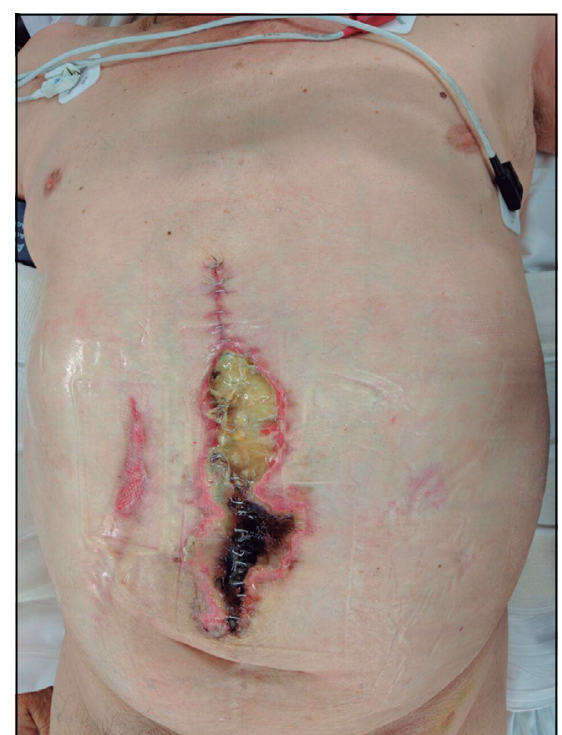

Figura 11. P1 Necrosis parcial de los colgajos en la línea media.

la resistencia a la infección, la biocompatibilidad con el tejido humano, la tolerancia a la exposición cutánea y la estabilidad mecánica. Además, estos productos tienen menor potencial de adherencia intestinal y de desarrollo de infección, siendo la mejor alternativa a las mallas sintéticas en reparaciones recurrentes o complicadas. Por tales razones, se han convertido en una opción popular y confiable para la reparación de la hernia durante los últimos años. Debido a la falta de ensayos comparativos más grandes no hay evidencia de qué malla biológica es mejor ${ }^{5}$. Las desventajas son su alto costo y la falta de estudios de seguimiento a largo plazo para la validación de su uso.

Materiales como la dermis humana acelular (AlloDerm, LifeCell, Branchburg, Nueva Jersey), dermis acelular porcina como Permacol (Covidien) y Strattice (LifeCell), y submucosa intestinal porcina (Biodesign, Cook Surgical Incorporated, Bloomington, Indiana) son los biomateriales más comúnmente usados $^{20}$.

En nuestro país no contábamos con estos productos. Hace algún tiempo se incorporó la malla biológica Strattice ${ }^{\circledR}$ a nuestro mercado, pero debido a su alto costo, no ha sido posible su uso en reconstrucción de pared abdominal para uso público. Ante la necesidad de resolver la problemática presentada en nuestros pacientes con eventraciones gigantes, es que desarrollamos la técnica descrita en este trabajo. Ocupamos la vaina anterior del recto abdominal como "malla biológica" para cerrar completamente los defectos herniarios, reduciendo el riesgo de fístulas entero-cutáneas e infecciones asociadas al uso de mallas sintéticas. Se trata de una técnica confiable, segura y reproducible.

\section{Procedimientos de contorno en combinación con la reparación de la hernia}

Frecuentemente hernias ventrales producen un excedente cutáneo importante luego de la reparación, lo que, sumado a las cicatrices, hacen necesario evaluar la calidad de la piel en pos de evitar complicaciones como necrosis cutánea e infecciones. Lograr un cierre de la piel y un contorno corporal adecuados también son aspectos muy importantes que considerar, pudiéndose realizar estos procedimientos en el momento de la reparación de la hernia ${ }^{21}$.

Algunos reportes en grupos de pacientes obesos mórbidos con importante baja de peso después de un procedimiento bariátrico y que, concomitantemente tenían una hernia incisional, sugieren que la reparación de la hernia y abdominoplastía puede realizarse simultáneamente con resultados aceptables $^{22}$. Este procedimiento es el más apropiado para los pacientes que ya están en su peso ideal de manera sostenida.

\section{Conclusión}

Esta modificación técnica es segura, útil y accesible para los pacientes con eventraciones gigantes. La tasa de complicaciones es baja y está dada, principalmente, por problemas relacionados al sufrimiento de los colgajos cutáneos.

Recomendamos el uso de mallas sintéticas de polipropileno para reforzar la pared abdominal. $\mathrm{Al}$ quedar estas en contacto sobre el plano muscular, su incorporación es más rápida, evitando así su exposición ante un eventual defecto por la pérdida de cobertura cutánea.

\section{Responsabilidades éticas}

Protección de personas y animales. Los autores declaran que para esta investigación no se han realizado experimentos en seres humanos ni en animales.

Confidencialidad de los datos. Los autores declaran que en este artículo no aparecen datos de pacientes.

Conflictos de interés: no hay. 


\section{Bibliografía}

1. Lechaux JP, Lechaux D, Chevrel JP. Traitement des éventrations de la paroi abdominale. Editions Techniques-Encycl Méd Chir, Techniques chirurgicales Appareil digestif. 2004. 40-165, 14p.

2. De Santis L, Frigo F, Bruttocao A, Terranova O. Pathophysiology of giant incisional hernias with loss of abdominal wall substance. Acta Biomed. 2003;74 Suppl 2:34-7.

3. Butler CE, Campbell KT. Minimally invasive component separation with inlay bioprosthetic mesh (MICSIB) for complex abdominal wall reconstruction. Plast Reconstr Surg. 2011;128:698-709.

4. Burger JW, Luijendijk RW, Hop WC, Halm JA, Verdaasdonk EG, Jeekel J. Long-term follow-up of a randomized controlled trial of suture versus mesh repair of incisional hernia. Ann Surg. 2004;240:578-83.

5. Beale EW, Hoxworth RE, Livingston EH, Trussler AP. The role of biologic mesh in abdominal wall reconstruction: a systematic review of the current literature. Am J Surg. 2012;204:510-7.

6. Blatnik J, Jin J, Rosen M. Abdominal hernia repair with bridging acellular dermal matrix-an expensive hernia sac. Am J Surg. 2008;196:47-50.

7. Smart NJ, Marshall M, Daniels IR. Biological meshes: a review of their use in abdominal wall hernia repairs. Surgeon 2012;10:159-71.

8. Hsu PW, Salgado CJ, Kent K, Finnegan M, Pello M, Simons R, et al. Evaluation of porcine dermal collagen (Permacol) used in abdominal wall. J Plast Reconstr Aesthet Surg. 2009;62:1484-9. DOI: 10.1016/j.bjps.2008.04.060. Epub 2008 Aug 20.

9. Höer J, Lawong G, Klinge U, Schumpelick V. Factors influencing the development of incisional hernia. A retrospective study of 2,983 laparotomy patients over a period of 10 years. Chirurg. 2002;73:474-80.

10. Klinge U, Si ZY, Zheng H, Schumpelick $\mathrm{V}$, Bhardwaj RS, Klosterhalfen B. Abnormal collagen I to III distribution in the skin of patients with incisional hernia. Eur Surg Res. 2000;32:43-8.

11. Young D Repair of epigastric incisional hernia. Br J Surg. 1961;48:514-6.

12. Ramírez OM, Ruas E, Dellon AL. "Components separation" method for closure of abdominal-wall defects: an anatomic and clinical study. Plast Reconstr Surg. 1990;86:519-26.

13. Shestak KC, Edington HJ, Johnson RR. The separation of anatomic components technique for the reconstruction of massive midline abdominal wall defects: anatomy, surgical technique, applications, and limitations revisited. Plast Reconstr Surg. 2000;105:731-8.

14. den Hartog D, Dur AH, Tuinebreijer WE, Kreis RW. Open surgical procedures for incisional hernias. Cochrane Database Syst Rev. 2008;(3):CD006438. DOI: 10.1002/14651858.CD006438.pub2.

15. de Vries Reilingh TS, van Goor H, Rosman C, Bemelmans MH, de Jong D, van Nieuwenhoven EJ, et al. "Components separation technique" for the repair of large abdominal wall hernias.
J Am Coll Surg. 2003;196:32-7.

16. de Vries Reilingh TS, van Goor H, Charbon JA, Rosman C, Hesselink EJ, et al. Repair of giant midline abdominal wall hernias: "components separation technique" versus prosthetic repair: interim analysis of a randomized controlled trial. World J Surg. 2007;31:756-63.

17. Ghali S, Turza KC, Baumann DP, Butler CE. Minimally invasive component separation results in fewer wound-healing complications than open component separation for large ventral hernia repairs. J Am Coll Surg. 2012;214:981-9.

18. Rosen MJ, Jin J, McGee MF, Williams C, Marks J, Ponsky JL. Laparoscopic component separation in the single-stage treatment of infected abdominal wall prosthetic removal. Hernia. 2007;11:43540.

19. Lowe JB, Garza JR, Bowman JL, Rohrich RJ, Strodel WE. Endoscopically assisted "components separation" for closure of abdominal wall defects. Plast Reconstr Surg. 2000;105:720-9.

20. Flurry MD, Michelotti BF, Moyer KE. Pedicled rectus femoris flap for coverage of complex open pelvic fractures. J Plast Reconstr Aesthet Surg. 2011;64:1490-4.

21. Mazzocchi M, Dessy LA, Ranno R, Carlesimo B, Rubino C. "Component separation" technique and panniculectomy for repair of incisional hernia. Am J Surg. 2011;201:776-83.

22. Shermak MA. Hernia repair and abdominoplasty in gastric bypass patients. Plast Reconstr Surg. 2006;117:1145-50; discussion 1151-2. 